\title{
Assessing allocation of responsibility for health management in pediatric liver transplant recipients
}

\begin{abstract}
Bilhartz JL, Lopez MJ, Magee JC, Shieck VL, Eder SJ, Fredericks EM. (2015) Assessing allocation of responsibility for health management in pediatric liver transplant recipients. Pediatr Transplant, 19: 538-546. DOI: $10.1111 /$ petr.12466.
\end{abstract}

Abstract: Given the increased risk for non-adherence and poor health outcomes in late adolescence, there is a need for better methods to evaluate and improve the transition process as adolescent patients are prepared to be independent adults. This study assessed the psychometrics and concurrent validity of a newly developed measure of AoR for health management in pediatric liver transplant patients. A total of 48 patients and 37 parents completed a 13-item measure of AoR. We performed an exploratory PCA on survey results and used component scores to assess the relationship between AoR and age, age at transplant, adherence, and health outcomes. Two primary components were identified: communication with the healthcare system and self-management tasks. Parent perception of adolescent responsibility for tasks related to communicating with the healthcare system was correlated, in younger patients, with increased nonadherence while responsibility for tasks related to self-management was correlated, in older patients, with decreased non-adherence. These results support AoR as a two-domain construct, and they provide targets for monitoring and intervention as adolescent patients advance toward transfer.

\author{
Jacob L. Bilhartz ${ }^{1}$, M. James Lopez ${ }^{1}$, \\ John C. Magee ${ }^{2,3}$, Victoria L. Shieck ${ }^{3}$, \\ Sally J. Eder ${ }^{1}$ and Emily M. Fredericks ${ }^{1,4}$ \\ ${ }^{1}$ Department of Pediatrics, University of Michigan \\ Health System, Ann Arbor, MI, USA, ${ }^{2}$ Department of \\ Surgery, University of Michigan Health System, Ann \\ Arbor, MI, USA, ${ }^{3}$ Transplant Center, University of \\ Michigan Health System, Ann Arbor, MI, USA, ${ }^{4}$ Child \\ Health Evaluation and Research (CHEAR) Unit, \\ University of Michigan Health System, Ann Arbor, \\ MI, USA \\ Key words: transition to adult care - adolescent \\ - liver transplantation - pediatrics - self-care \\ Jacob L. Bilhartz, 2015 Uppergate Drive NE, ECC 2nd \\ Floor, Atlanta, GA 30322, USA \\ Tel.: 404-785-1807 \\ Fax: 404-785-9017 \\ E-mail: jbilhar@emory.edu \\ Accepted for publication 9 March 2015
}

As long-term survival rates for pediatric liver transplant recipients continue to improve $(1,2)$, providers are faced with increasing numbers of patients reaching a period during which their care will ultimately be transferred to an adultbased clinic. This transfer is an experience that has been acknowledged as challenging by patients, families, and physicians alike (3-5), and the adolescent developmental period leading up to it is one regarded as high risk across all populations, both with and without the additional burden of a chronic health condition such as a transplant $(6,7)$. Thus, there is a critical need to develop a richer understanding of the process of transition, best appreciated as one that begins

\footnotetext{
Abbreviations: AoR, allocation of responsibility; OTIS, organ transplant information system; PCA, principal components analysis; s.d., standard deviation; tacro s.d., tacrolimus standard deviation; TRS, transition readiness survey.
}

long before, and in some cases continues long after, the physical transfer to a different clinic (5). Using a shared management model, the first stage of the transition process has been proposed to begin around $10 \mathrm{yr}$ of age, suggesting this as a time when providers should begin to introduce the concept of transition $(8,9)$. This is the age around which many children begin to develop some engagement with their own care (8). Over the subsequent years, attention to age-appropriate developmental milestones and the gradual shift in supervision and responsibility become a critical part of preparing these young children to become independent adults (8-11).

Increasing responsibility for different aspects of their health management has been identified as an issue about which adolescent patients have a particular interest (4). AoR refers to the degree of involvement of the adolescent, parent, and other caretakers in various aspects of disease management, and an appropriate shift in AoR 
over time is one of the components of a successful transition process $(9,10,12,13)$. A consensus conference sponsored by the Pediatric Committee of the American Society of Transplantation recommended that, by the time of transfer, adolescents and young adults should have successfully taken on responsibility for their health care in areas such as knowing names and doses of their medications, calling for their own prescription refills, maintaining a pill box, independently managing their appointments and communicating with their medical providers, and being able to seek urgent medical attention when appropriate (14). Despite these recommendations, there are relatively limited objective data on AoR and strategies for effectively shifting responsibility from parent to adolescent.

In the pediatric transplant population, age is associated with increased patient responsibility for health management $(10,15,16)$. Older adolescents/young adults are also known to be at higher risk for non-adherence to their recommended treatment regimen $(17,18)$. Yet, a direct relationship between AoR and risk for nonadherence has not been well established. Among pediatric kidney transplant recipients, increased patient-parent disagreement regarding AoR was significantly associated with medication nonadherence as measured by electronic medication monitoring devices (MEMS ${ }^{\circledR}$ TrackCaps) (15); however, the relationship between AoR and adherence has not yet been specifically investigated in pediatric liver transplant recipients.

The primary objective of this study was to assess the psychometric properties of a clinically derived measure of AoR in a population of pediatric liver transplant recipients. The measure described herein was developed as part of a larger quality improvement initiative within our center (10) to address a gap in the literature with respect to the assessment of AoR in transplant recipients. Since the initiation of our project, two additional measures of AoR have been published $(15,16)$, supporting the need to develop strategies to assess responsibility for health-related tasks among adolescents. Secondary aims of this study were to investigate the relationship between AoR and patient age, gender, and adherence as assessed by s.d. of consecutive, trough immunosuppressant blood levels $(8,19$ 21 ), and rate of clinic attendance. We hypothesized that adolescent liver transplant recipients will have better adherence and more favorable outcomes when responsibility for health management is shared or allocated to their guardians rather than when adolescents take primary responsibility for their care.

\section{Participants and methods}

Participants

Participants in this study were patients in our institution's pediatric liver transplant clinic who completed a measure of AoR as part of an ongoing quality improvement project centered on the process of transition $(4,10)$. Inclusion criteria were (i) $\geq 11 \mathrm{yr}$ of age (8), (ii) $>1 \mathrm{yr}$ from transplant and, thus, at less risk for iatrogenic fluctuations in their immunosuppressant levels, (iii) on tacrolimus as the primary immunosuppressant during the 12 months prior to or six months after completing the survey, and (iv) not a multiorgan transplant recipient. We recorded patient gender, age at the time of completing the measure, age at the time of transplant, time elapsed since transplant, and indication for transplant. This study was conducted from June of 2010 through November of 2013, and the "study period" for each individual participant included six months post-survey completion. This study was reviewed by our institutional review board and was determined to be "not regulated" due to its nature as a clinical quality improvement project.

\section{Measure of AoR}

As part of an ongoing clinical quality improvement project within our liver transplant program, all patients who are $\geq 11$ yr of age and at least six months from transplant complete a TRS biannually to guide transition planning. Based on results from an analysis of our initial TRS as well as a follow-up study of patient and parent perceptions of the transition process $(4,10)$, a separate AoR measure was added to the TRS in 2010. Items were developed through clinical observation, discussion among our institution's pediatric liver transplant team, and a review of the literature on AoR, transition, and adherence $(14,22,23)$. Our team is composed of a pediatric transplant hepatologist, transplant surgeon, transplant nurse coordinator, pediatric psychologist, social worker, and a dietician. The AoR measure consists of 13 items (Table 1). Adolescents and parents/ guardians (when available) answer identical questions on parallel self-report measures. Respondents were asked to choose from one of the three answers: (i) parent/guardian takes primary responsibility, (ii) responsibility is shared between the parent/guardian and the adolescent, or (iii) adolescent takes primary responsibility. Administration time is $<5$ min during the course of a routine clinic visit. Items with the parent or guardian assuming primary responsibility were given a score of 1 , and those with the adolescent sharing or taking primary responsibility were scored as 2 and 3, respectively. Thus, higher scores are indicative of greater responsibility allocated to the adolescent.

\section{Assessment of adherence}

\section{Immunosuppressant variability}

Fluctuation in immunosuppressant trough levels over time, as measured by s.d., has been directly correlated with rejection of the transplant in both pediatric and adult patients $(8,19-21)$. Shown to have a stronger relationship with rejection than either patient or provider assessments of adherence (8), immunosuppressant variability thus presents itself as a surrogate marker for non-adherence and can be calculated from laboratory values obtained in the course of routine clinical care. To assess the risk for non-adherence in this manner, we retrieved serial tacrolimus trough levels for 
Bilhartz et al.

Table 1. AoR survey items

Survey item no.

\begin{tabular}{cl}
\hline 1 & Who remembers morning medications? \\
2 & Who remembers evening medications? \\
3 & Who makes sure medications are taken properly? \\
4 & Who makes sure there is enough medication? \\
5 & Who calls in prescription refills? \\
6 & Who organizes medications? (Like in a pillbox) \\
7 & Who remembers appointment times? \\
8 & Who remembers laboratory draws? \\
9 & Who makes clinic appointments? \\
10 & Who discusses health needs at clinic visits? \\
11 & Who notices health changes? \\
12 & Who calls the medical team with health-related questions? \\
13 & Who describes health condition to others? \\
\hline
\end{tabular}

participants from their electronic medical record and our institution's OTIS. Laboratory values obtained while inpatient were omitted. Standard deviations were calculated for consecutive tacrolimus trough levels for all participants for the study period of six months following their completion of our AoR measure. To guard against the introduction of bias, laboratory values were retrieved from the medical record and placed into an electronic database by research assistants not directly involved in clinical care. We used a tacro s.d. of two as a cutoff for non-adherence (19). In addition to immunosuppressant variability as a measure of non-adherence, we also retrieved each participant's individual target tacrolimus range from their electronic medical record and calculated the percentage of trough levels out of the target range during the study period. At our center, patients $>12$ months post-transplant obtain laboratory testing every two months, unless otherwise medically indicated.

\section{Clinic attendance}

To assess non-medication-related aspects of regimen adherence, we also examined patterns of clinic attendance. The number of visits missed without re-scheduling (i.e., "noshow") was collected for each participant during the study period, as was the percentage of visits attended as scheduled.

\section{Health outcomes}

The number of days hospitalized for rejection during the study period was recorded. We also recorded the number of episodes of biopsy-proven rejection during the study period.

\section{Data analysis}

To identify potential components underlying this AoR measure, we conducted an exploratory PCA on the 13 items of the adolescent and the parent surveys separately. PCA with a varimax rotation was performed to maximize the unique nature of each component extracted (24). Internal consistency of the complete 13-item measure, as well as for each of the resulting components, was assessed using Cronbach's alpha. Component scores were calculated for each participant, which reflected to what degree they were represented by each component identified. The degree of component saturation was used as a means of evaluating the stability of our PCA (25).
The normality of the distribution of AoR survey responses, adherence measures, and health outcome variables was assessed. We looked for any differences between participants and non-participants (see Table 2 for reasons for non-participation) in terms of their gender, demographics, adherence measures, and health outcome variables using either the Mann-Whitney or chi-square test, as statistically appropriate. Additionally, again using either the MannWhitney or chi-square test, we looked for differences between the genders in terms of their demographics, adherence measures, health outcome variables, and survey responses. Adolescent-parent agreement was assessed using the Wilcoxon signed-rank test to compare responses on each of the 13 items for adolescents and parents.

Based on the non-normal distribution of survey responses, we used Spearman's rank correlation coefficient (rho) to assess the relationship between age, age at and time since transplant, AoR, adherence, and health outcomes. Partial correlations were used to control for the effect of non-adherence on the relationship between AoR and age. To minimize the complications arising from multiple comparisons with a 13-item measure, we used the component scores resulting from our PCA as variables reflecting different aspects of AoR for each participant when performing the analyses. Given the exploratory nature of this study and the aim to identify targets for future work at the expense of potential initial false positives, we did not apply any further corrections for multiple comparisons. All p-values reported in this study are two-tailed.

The analyses for this study were conducted using SPSS for Windows (version 20; IBM Corporation, Armonk, NY, USA).

\section{Results}

Participants

From June of 2010 through November of 2013, 83 patients participated in our institution's pediatric liver transplant transition program. Of those, 48 met study inclusion criteria, completed

\begin{tabular}{|c|c|}
\hline \multirow{2}{*}{\multicolumn{2}{|c|}{$\begin{array}{l}N=48 \\
\text { Gender (\%) }\end{array}$}} \\
\hline & \\
\hline \multicolumn{2}{|l|}{ Male } \\
\hline \multicolumn{2}{|l|}{ Female } \\
\hline \multicolumn{2}{|l|}{ Mean age (s.d.) } \\
\hline \multicolumn{2}{|l|}{ Mean age at transplant (s.d.) } \\
\hline \multicolumn{2}{|l|}{ Mean time since transplant (s.d.) } \\
\hline \multicolumn{2}{|l|}{ Non-adherent by Tacro s.d. >2 (\%) } \\
\hline \multicolumn{2}{|l|}{$\begin{array}{l}\text { Total episodes of rejection in all } \\
\text { patients in this study }\end{array}$} \\
\hline \multicolumn{2}{|c|}{ Reasons for non-participation (from initial sample of 83 patients) } \\
\hline Reason & Number of patients (\%) \\
\hline Less than 1 yr since transplant at survey completion & $11(13)$ \\
\hline $\begin{array}{l}\text { Lost to follow up or data otherwise unavailable } \\
\text { during the study period }\end{array}$ & $10(12)$ \\
\hline AoR measure not completed & $7(8.4)$ \\
\hline $\begin{array}{l}\text { On a primary immunosuppressant other than } \\
\text { tacrolimus during the study period or year prior }\end{array}$ & $6(7.2)$ \\
\hline Multiorgan transplant recipient & $1(1.2)$ \\
\hline
\end{tabular}


the AoR portion of the TRS, and had the requisite clinical data available (see Table 2 for demographics and reasons for study non-participation). Thirty-seven (77\%) of those 48 patients had parent or guardian data available as well. Mean age was $15.8 \mathrm{yr}$ with a range from 11.3 to $20.1 \mathrm{yr}$ (Fig. 1), and mean age at transplant was $6.37 \mathrm{yr}$ with a range from six months to $11.4 \mathrm{yr}$. Fifty percent of the participants in this study underwent liver transplantation due to biliary atresia, with other recurrent diagnoses including idiopathic acute liver failure $(14.6 \%)$, autoimmune hepatitis $(10.4 \%)$, and hepatoblastoma $(6.3 \%)$. There were no statistically significant differences between participants and non-participants in terms of their gender, stage in the transition process (early being ages 10-12 yr, middle 13-15 yr, and late $16 \mathrm{yr}$ and older) (10), time since transplant, indication for transplant, occurrence of rejection, appointment no-shows, or rate of clinic attendance ( $p$-values from 0.066 to 0.76 ). Likewise, there were no differences between the male and female patients in our sample in terms of their stage in the transition process, indication for transplant, time since transplant, non-adherence as defined by tacro s.d. $>2$, percentage of laboratory values out of goal range, appointment no-shows, or rate of clinic attendance ( $\mathrm{p}$-values from 0.16 to $0.95)$.

Psychometric properties of AoR measure

Patient responses

A PCA was performed on the 13 survey items. Using Cattell's elbow criteria, two components were extracted $(24,26)$. Together, these components accounted for $64.2 \%$ of the total

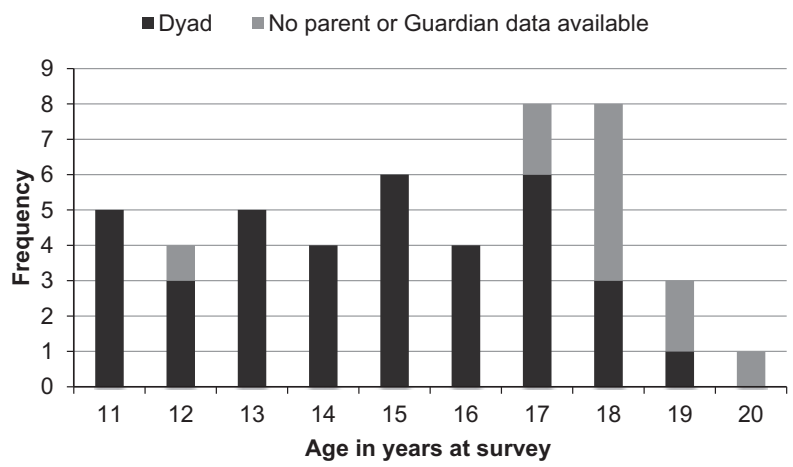

Fig. 1. Distribution of participant ages as well as the availability of parent/guardian data. In all, 48 patients met our inclusion criteria. A total of 37 of those patients also had data available from a parent or guardian, and the majority of those without parent data were the older patients. Mean age at the time of survey completion was $15.8 \mathrm{yr}$ with a range from 11.3 to 20.1 . variance. Varimax rotation was then performed on the component loadings. The component saturation in these results, with both components having $>4$ loadings of $>0.6$, indicated that the identified components were stable (25). Given the exploratory nature of this study, we closely examined which component each item influenced more strongly - a relationship represented by the rotated component loadings shown in Table 3. Component 1 included items involving communicating with different aspects of the healthcare system, whereas component 2 included items pertaining to self-management. Component 1 communication tasks - had an internal consistency (Cronbach's alpha) of 0.92, and component 2 - self-management tasks - had an internal consistency of 0.88 . The internal consistency of the complete 13-item measure was also strong at 0.93 , and individual item alphas across the complete measure are listed in Table 3 (range 0.92-0.93).

\section{Parent responses}

Similar to the adolescent responses, a PCA was performed on the 13 parent survey items with a varimax rotation. Again, two components were extracted, and together, these two components accounted for $57.8 \%$ of the total variance. Rotated component loadings for parent respondents are shown in Table 4. Again, $\geq 4$ loadings of $>0.6$ for each component indicated stability in the components we identified (25). We again examined which component each of the 13 items influenced more strongly. Component 1 items again involved communicating with the

Table 3. Two components identified for patient AoR surveys

\begin{tabular}{|c|c|c|c|}
\hline \multirow[b]{2}{*}{$\begin{array}{l}\text { AoR survey } \\
\text { item }\end{array}$} & \multicolumn{2}{|c|}{ Rotated component loading* } & \multirow[b]{2}{*}{ Cronbach's $\alpha$} \\
\hline & $\begin{array}{l}\text { Component } 1 \\
\text { Communication tasks }\end{array}$ & $\begin{array}{l}\text { Component } 2 \\
\text { Self-management } \\
\text { tasks }\end{array}$ & \\
\hline 1 & 0.13 & 0.80 & 0.93 \\
\hline 2 & 0.21 & 0.76 & 0.93 \\
\hline 3 & 0.24 & 0.81 & 0.93 \\
\hline 4 & 0.40 & 0.75 & 0.92 \\
\hline 5 & 0.84 & 0.27 & 0.92 \\
\hline 6 & 0.25 & 0.75 & 0.93 \\
\hline 7 & 0.65 & 0.41 & 0.93 \\
\hline 8 & 0.76 & 0.33 & 0.93 \\
\hline 9 & 0.88 & 0.089 & 0.93 \\
\hline 10 & 0.72 & 0.35 & 0.93 \\
\hline 11 & 0.29 & 0.52 & 0.93 \\
\hline 12 & 0.78 & 0.21 & 0.93 \\
\hline 13 & 0.52 & 0.50 & 0.93 \\
\hline
\end{tabular}

*Number reflecting how strongly each item impacted on the identified components

Bold text indicates to which component each item was assigned.. 
Table 4. Two components identified for parent AoR surveys

\begin{tabular}{llll}
\hline & \multicolumn{2}{l}{ Rotated component loading* } & \\
\cline { 2 - 3 } $\begin{array}{lll}\text { AoR survey } \\
\text { item }\end{array}$ & $\begin{array}{lll}\text { Component 1 } \\
\text { Communication tasks }\end{array}$ & $\begin{array}{l}\text { Component 2 } \\
\text { Self-management tasks }\end{array}$ & Cronbach's $\boldsymbol{\alpha}$ \\
\hline 1 & 0.22 & $\mathbf{0 . 8 1}$ & 0.86 \\
2 & 0.21 & $\mathbf{0 . 7 9}$ & 0.87 \\
3 & -0.18 & $\mathbf{0 . 8 3}$ & 0.88 \\
4 & 0.37 & $\mathbf{0 . 7 0}$ & 0.86 \\
5 & $\mathbf{0 . 7 2}$ & 0.26 & 0.85 \\
6 & 0.46 & $\mathbf{0 . 4 7}$ & 0.87 \\
7 & $\mathbf{0 . 6 8}$ & 0.24 & 0.87 \\
8 & $\mathbf{0 . 8 4}$ & 0.15 & 0.86 \\
9 & $\mathbf{0 . 7 0}$ & 0.32 & 0.87 \\
10 & $\mathbf{0 . 5 1}$ & 0.25 & 0.87 \\
11 & $\mathbf{0 . 5 3}$ & 0.34 & 0.87 \\
12 & $\mathbf{0 . 8 0}$ & -0.023 & 0.87 \\
13 & $\mathbf{0 . 7 6}$ & -0.086 & 0.88 \\
\hline
\end{tabular}

*Number reflecting how strongly each item impacted on the identified components.

Bold text indicates to which component each item was assigned.

healthcare system; however, "Who notices health changes" loaded more strongly on component 1 with the parent surveys as opposed to with the adolescent. Component 2 comprised items pertaining to self-management or awareness. Communication tasks for the parent surveys had a Cronbach's alpha of 0.86 , and self-management tasks had a Cronbach's alpha of 0.81. As with the adolescent surveys, the internal consistency of the complete 13-item parent measure was strong with a Cronbach's alpha of 0.88 and a range from 0.85 to 0.88 (see Table 4 ).

\section{Adolescent and parent agreement}

A Wilcoxon signed-rank test demonstrated that there was not a statistically significant difference between adolescent and parent responses on any of the 13 AoR survey items ( $p$-values from 0.058 to 1.0) except for \#13 - "Who describes health conditions to others?" - on which adolescents had a higher mean score of 1.97 compared to parents at $1.75(\mathrm{p}$-value $=0.039)$, indicating that adolescents saw themselves as more responsible than their parents did for describing their condition to others.

\section{AoR and gender}

There were no statistically significant differences between male and female patients for adolescent report of self-management tasks, or for parent report of either AoR domain. However, there was a significant difference between male and female adolescent-reported responsibility for communication tasks $(-0.48$ and 0.29 , respec- tively; Mann-Whitney p-value $=0.035$ ), indicating that female adolescents perceive themselves as more responsible for communication tasks.

AoR and age

Age at time of survey completion was positively correlated with adolescent report of responsibility both for communication tasks (rho $=0.40$, $\mathrm{p}=0.012$ ) and for self-management tasks (rho $=0.52, \mathrm{p}=0.001$ ), suggesting more perceived responsibility with increasing chronological age. Among parent reports, adolescent age was positively correlated with increased responsibility for self-management tasks (rho $=0.39$, $\mathrm{p}=0.029$ ), but not with communicating with the healthcare system $(p=0.082)$. Correlations between age and AoR remained significant when controlling for non-adherence (i.e., tacro s.d.).

Age, adherence, and health outcomes

Non-adherence as measured by tacro s.d. was positively correlated with increased age in the 48 patients included in this study (rho $=0.36$, $\mathrm{p}=0.011)$. Age at transplant and time since transplant were not significantly related to tacro s.d. $(\mathrm{p}=0.13$ and 0.82 , respectively). Sixteen $(33.3 \%)$ of the patients in this study were nonadherent as defined by a tacro s.d. $>2$.

Important clinical outcomes such as number of days hospitalized for rejection (rho $=0.32$, $\mathrm{p}=0.025)$ and episodes of biopsy-proven rejection ( $\mathrm{rho}=0.38, \mathrm{p}=0.007$ ) were also positively correlated with tacro s.d. However, the overall incidence of rejection throughout this sample was low with only three episodes during the study period. There were no statistically significant correlations noted between either adolescent or parent reports of AoR and health outcomes such as hospital days and occurrence of rejection ( $p$-values from 0.12 to 0.34 ).

\section{AoR and adherence}

Adolescent reports of AoR were not significantly correlated with any of the adherence measures in this study (p-values from 0.22 to 0.99 ). For those patients in the early phase of transition (see Table 5), parent report of adolescent responsibility for communication tasks was significantly correlated with the percentage of laboratory values out of goal range ( $\%$ OOR), but not with other measures of adherence. In the middle phase of transition (Table 5), reports obtained from parents indicated that adolescent responsibility for communication tasks was significantly correlated with a decreased rate of clinic attendance. 
Table 5. Correlations between AoR and adherence in the different phases of transition

\begin{tabular}{|c|c|c|c|c|c|c|}
\hline & \multicolumn{2}{|l|}{$\begin{array}{l}\text { Early transition } \\
10-12 \text { yr }\end{array}$} & \multicolumn{2}{|l|}{$\begin{array}{l}\text { Mid-transition } \\
13-15 \mathrm{yr}\end{array}$} & \multicolumn{2}{|l|}{$\begin{array}{l}\text { Late transition } \\
\geq 16 \mathrm{yr}\end{array}$} \\
\hline & $\begin{array}{l}\text { Parent } \\
\text { communication }\end{array}$ & Parent self-management & Parent communication & Parent self-management & Parent communication & Parent self-management \\
\hline Tacro s.d. & $\begin{array}{l}\text { rho }=0.21 \\
p=0.65\end{array}$ & $\begin{array}{l}\text { rho }=-0.68 \\
p=0.094\end{array}$ & $\begin{array}{l}\text { rho }=0.36 \\
p=0.23\end{array}$ & $\begin{array}{l}\text { rho }=-\mathbf{0 . 6 0} \\
p=\mathbf{0 . 0 3 1}\end{array}$ & $\begin{array}{l}\text { rho }=0.58 \\
p=0.060\end{array}$ & $\begin{array}{l}\text { rho }=-0.091 \\
p=0.79\end{array}$ \\
\hline$\%$ OOR & $\begin{array}{l}\text { rho }=\mathbf{0 . 8 9} \\
p=\mathbf{0 . 0 0 7}\end{array}$ & $\begin{array}{l}\text { rho }=-0.75 \\
p=0.052\end{array}$ & $\begin{array}{l}\text { rho }=0.11 \\
p=0.72\end{array}$ & $\begin{array}{l}\text { rho }=-\mathbf{0 . 6 5} \\
p=\mathbf{0 . 0 1 6}\end{array}$ & $\begin{array}{l}\text { rho }=0.28 \\
p=0.40\end{array}$ & $\begin{array}{l}\text { rho }=-0.16 \\
p=0.63\end{array}$ \\
\hline No-shows & $\begin{array}{l}\text { rho }=0.58 \\
p=0.17\end{array}$ & $\begin{array}{l}\text { rho }=-0.54 \\
p=0.23\end{array}$ & $\begin{array}{l}\text { rho }=0.46 \\
p=0.12\end{array}$ & $\begin{array}{l}\text { rho }=-0.57 \\
p=0.042\end{array}$ & $\begin{array}{l}\text { rho }=-0.30 \\
p=0.37\end{array}$ & $\begin{array}{l}\text { rho }=-\mathbf{0 . 6 1} \\
\mathrm{p}=\mathbf{0 . 0 4 7}\end{array}$ \\
\hline$\%$ Attendance & $\begin{array}{l}\text { rho }=-0.62 \\
p=0.14\end{array}$ & $\begin{array}{l}\text { rho }=0.45 \\
p=0.31\end{array}$ & $\begin{array}{l}\text { rho }=-0.57 \\
p=0.042\end{array}$ & $\begin{array}{l}\text { rho }=0.19 \\
p=0.53\end{array}$ & $\begin{array}{l}\text { rho }=0.21 \\
p=0.54\end{array}$ & $\begin{array}{l}\text { rho }=0.54 \\
p=0.087\end{array}$ \\
\hline
\end{tabular}

Tacro s.d. = tacrolimus standard deviation during the study period; $\% 00 \mathrm{R}=$ percent of tacrolimus levels out of goal range; no-shows = number of appointments missed; \% attendance $=$ percentage of appointments attended as scheduled.

Bold text indicates correlations that were statistically significant.

Parent-reported adolescent responsibility for self-management tasks was significantly correlated with a decrease in tacro s.d., \% OOR, and the number of appointment no-shows. Finally, for those patients in late transition (Table 5), parent reports of responsibility for self-management tasks were significantly correlated with a decreased number of appointment no-shows.

\section{Discussion}

The primary aim of this study was to assess the psychometric properties of a clinically derived measure of AoR for health management tasks in pediatric liver transplant recipients. Our data demonstrated that the 13 items comprising the adolescent measure had two primary underpinning components - communication tasks and self-management tasks. The two components of the parent survey were the same as those for the adolescents with one exception: The item "Who notices health changes?" loaded on the "communication tasks" domain for parents. This may reflect the need for some level of communication in order for parents to be aware of the health status changes that their child/adolescent has noticed. The PCA described in this study yields a useful framework for the development of future assessments of AoR and refinements of this measure, with a focus on communication and selfmanagement skills.

The two components of AoR in this study are similar to those identified in previous work assessing transition readiness (27). However, in the current study, we demonstrated a significant relationship between AoR and objective measures of adherence. Interestingly, parents' perception of their child's responsibility had a stronger relationship with adherence in this study than did the adolescents' own reporting, reinforcing the importance of collecting information regarding AoR from more than one source. The correlation between increased parent-reported adolescent responsibility for communication tasks (see Fig. 2) and increased non-adherence suggests that younger patients whose parents perceive them as being more responsible for these issues may be at higher risk for non-adherence and its associated complications and, thus, require closer monitoring by the medical team.
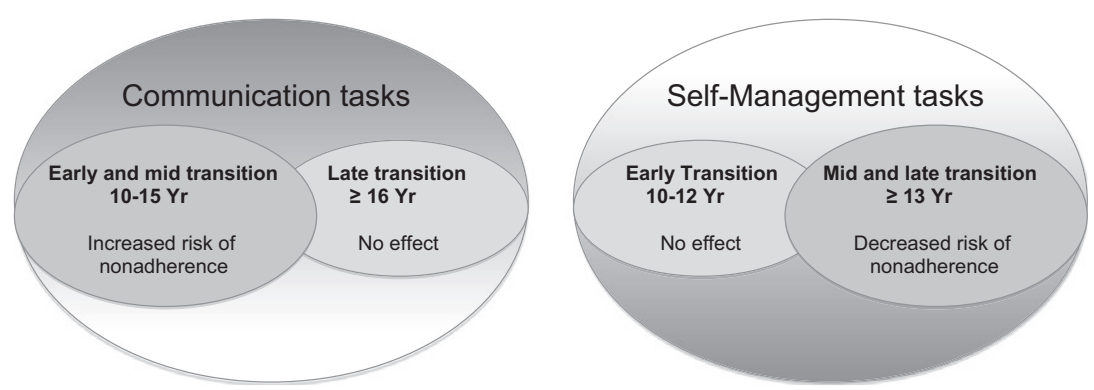

Fig. 2. The relationship between adherence and parent-reported AoR for communication and self-management tasks varies based on the stage of transition. Patients in early or middle transition whose parents perceived them as more responsible for communication tasks were at higher risk for non-adherence. Patients in middle or late transition whose parents perceived them as more responsible for self-management task were at a decreased risk of non-adherence. 
The correlation between increased parentreported adolescent responsibility for self-management tasks, and, in patients in mid-late transition, improved adherence (Fig. 2) suggests that appropriate transfer of AoR can be fostered and supported when patients are encouraged to develop self-management skills. Patients in late transition who have not yet begun to take primary responsibility for these issues are unlikely to be ready for transfer despite their older age.

This study demonstrated a significant relationship between older age and increased adolescent perception of responsibility for all aspects of their health care. This finding is consistent with previous reports on AoR in liver transplant recipients as well as in other chronic disease populations $(12,15,16,28)$. However, despite this increased perception of their own responsibility, older patients as a whole remained the most nonadherent, and their perception of responsibility did not translate into any measurable impact on adherence or health outcomes. These findings reinforce the importance of focusing on skills and knowledge rather than solely on age as the criterion to determine when an adolescent is ready for independent self-management.

The results of this study, while promising, must be viewed in the context of its limitations. This was a retrospective study undertaken as part of an ongoing clinical quality improvement project at a single pediatric transplant center, thus potentially limiting generalizability. Similarly, our sample size may have limited our study power, precluding us from identifying small, yet potentially significant, findings. The "study period" was a different chronologic six months for each patient, thus introducing potential confounding factors - such as variability in clinic personnel, variability in clinical practices, seasonal or environmental factors, or other unknown issues - that may have affected our results. The total number of comparisons in this study may have led to spurious findings. We opted to report $\mathrm{p}$-values and did not employ stringent correction for multiple comparisons, to include relationships that may have important clinical implications. The findings from the current study will provide the foundation for a larger, controlled study investigating predictors of adherence and transition readiness. Additionally, while there is value in assessing the degree of an adolescent's involvement in their own care to identify targets for intervention, this study does not offer insight into the overall success of the transition process - that is, continued adherence to medications and good health outcomes following transfer to an adult clinic. Thus, future work with this measure will include a prospective assessment of AoR, identification of the impact of different clinic-based interventions on AoR, and examination of the predictive value of adolescent AoR on clinically significant outcomes following the transfer to adult-based care.

Our hypothesis that adherence would be improved when more responsibility is allocated to the parents did not yield conclusive results, as the relationship between adherence and AoR was dependent on the source of the report (i.e., patient vs. parent) and the stage of transition. This study is part of a larger quality improvement initiative aimed at enhancing adherence and transition readiness. This use of an AoR measure was intended to guide clinical care for adolescent liver transplant recipients within our center. At the time of study initiation, no other measures of AoR were available; thus, this study did not aim to compare different measures. Nevertheless, the unique contributions of the PCA results described in this study add to growing literature supporting the concept of an AoR measure as a tool for identifying pediatric transplant recipients who are at risk for non-adherence and poor health outcomes. Given the recognized importance of a successful transition process across all pediatric chronic illness populations (3, 5,29 ), this measure could also be used with adolescents with other chronic health conditions, as the survey items are not restricted to liver transplantation. We will use the results from this study to revise our AoR measure and potentially expand its use to other transplant programs or disease populations.

The findings of the current study are being used to further investigate the role of AoR in the transition process to expand our knowledge of factors related to post-transfer success. There are a multitude of factors that influence self-management of chronic illness and medication adherence. In our ongoing quality improvement efforts, we seek to incorporate additional assessment strategies to determine the role of AoR, along with other factors including health literacy and social support, in promoting adherence and optimal health status during the transition to adult care. Our long-term goal is to identify specific predictors of a successful transfer as well as targets for intervention as patients progress toward that milestone.

\section{Conclusions}

This study demonstrated high reliability for a clinically derived 13-item measure of AoR. Each of the two underlying components also had 
excellent independent reliability. Knowledge of the underlying components representing responsibility for communication and self-management tasks should be used to guide understanding of AoR, as well as future revisions and ongoing use of this measure. While older patients perceived themselves to be more responsible for their care, that perceived responsibility did not correlate with any improved health outcomes in this study, and these older patients were also the most at risk for non-adherence. Given the correlations seen between parent reporting of adolescent responsibility and non-adherence, patients reported to be more responsible for communication tasks should be monitored closely especially at a younger age, and older patients should demonstrate independent responsibility for self-management tasks prior to transfer. While AoR is just one component of the transition process, these results reinforce its importance and potential utility as a way to determine, aside from looking at mere chronologic age, which of our patients may be ready to move on.

\section{Acknowledgments}

This work was supported by Charles Woodson Biostatistics Fund Grant G010598 to Dr. Bilhartz and NIH Grant K23DK090202 to Dr. Fredericks. We thank Dr. Trivellore Raghunathan and Dr. Kerby Shedden for their statistical consultation during the preparation of this manuscript.

\section{Authors' contributions}

Jacob L. Bilhartz contributed to concept/design, statistics, data analysis/interpretation, drafting of this manuscript, approval of the final manuscript, and securing of funding. M. James Lopez contributed to concept/design, critical revision of the manuscript, and approval of the final version. John C. Magee contributed to concept/design, critical revision of the manuscript, and approval of the final version. Victoria L. Shieck contributed to concept/design, data collection, critical revision of the manuscript, and approval of the final version. Sally J. Eder contributed to data concept/ design, data analysis and interpretation, critical revision of the manuscript, and approval of the final version. Emily M. Fredericks contributed to concept/design, statistics, data analysis/interpretation, critical revision of the manuscript, approval of the final version, and securing of funding.

\section{References}

1. Organ Procurement and Transplantation Network (OPTN) and Scientific Registry of Transplant Recipients (SRTR). OPTN/SRTR 2010 Annual Data Report. Rockville, MD: Department of Health and Human Services, Health Resources and Services Administration, Healthcare Systems Bureau, Division of Transplantation, 2011. [Internet].

2. KIM JJ, Marks SD. Long-term outcomes of children after solid organ transplantation. Clinics 2014: 69: 28-38.

3. Blum RW, Garell D, Hodgman CH, et al. Transition from child-centered to adult health-care systems for adolescents with chronic conditions - a position paper of the Society for Adolescent Medicine. J Adolesc Health 1993: 14: 570-576.

4. Fredericks EM, Dore-Stites D, Lopez MJ, et al. Transition of pediatric liver transplant recipients to adult care: Patient and parent perspectives. Pediatr Transplant 2011: 15: 414-424.

5. Sawyer SM, Blair S, Bowes G. Chronic illness in adolescents: Transfer or transition to adult services? J Paediatr Child Health 1997: 33: 88-90.

6. Park MJ, Paul Mulye T, Adams SH, Brindis CD, Irwin CE $J_{R}$. The health status of young adults in the United States. J Adolesc Health 2006: 39: 305-317.

7. Taylor R, Franck LS, Gibson F, Dhawan A. A critical review of the health-related quality of life of children and adolescents after liver transplantation. Liver Transpl 2005: 11: 51-60.

8. Shemesh E, Shneider BL, Savitzky JK, et al. Medication adherence in pediatric and adolescent liver transplant recipients. Pediatrics 2004: 113: 825-832.

9. Kieckhefer GM, Trahms CM. Supporting development of children with chronic conditions: From compliance toward shared management. Pediatr Nurs 2000: 26: 354-363.

10. Fredericks EM, Dore-Stites D, Well A, et al. Assessment of transition readiness skills and adherence in pediatric liver transplant recipients. Pediatr Transplant 2010: 14: 944-953.

11. Mickley KL, Burkhart PV, Sigler AN. Promoting normal development and self-efficacy in school-age children managing chronic conditions. Nurs Clin North Am 2013: 48: 319-328.

12. Walders N, Drotar D, Kercsmar C. The allocation of family responsibility for asthma management tasks in African-American adolescents. J Asthma 2000: 37: 89-99.

13. Martin S, Elliott-Desorbo DK, Wolters PL, et al. Patient, caregiver and regimen characteristics associated with adherence to highly active antiretroviral therapy among HIV-infected children and adolescents. Pediatr Infect Dis J 2007: 26: 61-67.

14. Bell Le, Bartosh SM, Davis CL, et al. Adolescent transition to adult care in solid organ transplantation: A consensus conference report. Am J Transplant 2008: 8: 2230-2242.

15. Pai Al, Gray E, Kurivial K, Ross J, Schoborg D, Goebel J. The allocation of treatment responsibility scale: A novel tool for assessing patient and caregiver management of pediatric medical treatment regimens. Pediatr Transplant 2010: 14: $993-$ 999.

16. Piering K, Arnon R, Miloh ta, Florman S, Kerkar N, AnNUNZIATO RA. Developmental and disease-related influences on self-management acquisition among pediatric liver transplant recipients. Pediatr Transplant 2011: 15: 819-826.

17. Sudan DL, Shaw BW JR, Langnas AN. Causes of late mortality in pediatric liver transplant recipients. Ann Surg 1998: 227: 289-295.

18. Annunziato RA, Emre S, Shneider B, Barton C, Dugan CA, SHEMESH E. Adherence and medical outcomes in pediatric liver transplant recipients who transition to adult services. Pediatr Transplant 2007: 11: 608-614.

19. Venkat VL, Nick TG, Wang Y, Bucuvalas JC. An objective measure to identify pediatric liver transplant recipients at risk for late allograft rejection related to non-adherence. Pediatr Transplant 2008: 12: 67-72.

20. Stuber Ml, Shemesh E, Seacord D, Washington J, HelleMANN G, MCDiarmid S. Evaluating non-adherence to immunosuppressant medications in pediatric liver transplant recipients. Pediatr Transplant 2008: 12: 284-288.

21. Supelana C, Annunziato RA, Schiano TD, et al. Medication level variability index predicts rejection, possibly due to nonadherence, in adult liver transplant recipients. Liver Transpl 2014: 20: 1168-1177.

22. McDonagh JE. Growing up and moving on: Transition from pediatric to adult care. Pediatr Transplant 2005: 9: 364-372. 


\section{Bilhartz et al.}

23. Paone MC, Wigle M, Saewyc E. The on trac model for transitional care of adolescents. Prog Transplant 2006: 16: 291-302.

24. Rummel RJ. Applied Factor Analysis. Evanston, IL: Northwestern University Press, 1970.

25. Guadagnoli E, Velicer WF. Relation of sample size to the stability of component patterns. Psychol Bull 1988: 103: 266275.

26. Cattell RB. The scree test for the number of factors. Multivar Behav Res 1966: 1: 245-276.
27. Sawicki GS, Lukens-Bull K, Yin X, et al. Measuring the transition readiness of youth with special healthcare needs: Validation of the traq transition readiness assessment questionnaire. J Pediatr Psychol 2011: 36: 160-171.

28. Anderson BJ, Auslander WF, Jung KC, Miller JP, SAntiago JV. Assessing family sharing of diabetes responsibilities. J Pediatr Psychol 1990: 15: 477-492.

29. SAWYer SM, Aroni RA. Self-management in adolescents with chronic illness. What does it mean and how can it be achieved? Med J Aust 2005: 183: 405-409. 